\title{
Article \\ Rice Transcription Factor OsWRKY55 Is Involved in the Drought Response and Regulation of Plant Growth
}

\author{
Kai Huang ${ }^{\dagger}$, Tao $\mathrm{Wu}^{\dagger}{ }^{\dagger}$, Ziming Ma, Zhao Li, Haoyuan Chen, Mingxing Zhang, Mingdi Bian, Huijiao Bai, \\ Wenzhu Jiang * and Xinglin Du*
}

check for

updates

Citation: Huang, K.; Wu, T.; Ma, Z.; Li, Z.; Chen, H.; Zhang, M.; Bian, M.; Bai, H.; Jiang, W.; Du, X. Rice Transcription Factor OsWRKY55 Is Involved in the Drought Response and Regulation of Plant Growth. Int. J. Mol. Sci. 2021, 22, 4337. https:// doi.org/10.3390/ijms22094337

Academic Editor: Kiyosumi Hori

Received: 18 March 2021

Accepted: 20 April 2021

Published: 21 April 2021

Publisher's Note: MDPI stays neutral with regard to jurisdictional claims in published maps and institutional affiliations.

Copyright: (c) 2021 by the authors. Licensee MDPI, Basel, Switzerland. This article is an open access article distributed under the terms and conditions of the Creative Commons Attribution (CC BY) license (https:// creativecommons.org/licenses/by/ $4.0 /)$.
Jilin Province Engineering Laboratory of Plant Genetic Improvement, College of Plant Science, Jilin University, Changchun 130062, China; 15584159829@163.com (K.H.); jidawt@jlu.edu.cn (T.W.); mzm1040722406@163.com (Z.M.); zklizhao@jlu.edu.cn (Z.L.); hychen18@mails.jlu.edu.cn (H.C.); mingxing19@jlu.edu.cn (M.Z.); bianmd@jlu.edu.cn (M.B.); bhj20212021@163.com (H.B.)

* Correspondence: jwz1975@jlu.edu.cn (W.J.); duxinglin2004@163.com (X.D.); Tel.: +86-25-87835716 (W.J.); $+86-25-87836251$ (X.D.)

+ These authors have contributed equally to this work.

\begin{abstract}
WRKY transcription factors (TFs) have been reported to respond to biotic and abiotic stresses and regulate plant growth and development. However, the molecular mechanisms of WRKY TFs involved in drought stress and regulating plant height in rice remain largely unknown. In this study, we found that transgenic rice lines overexpressing OsWRKY55 (OsWRKY55-OE) exhibited reduced drought resistance. The OsWRKY55-OE lines showed faster water loss and greater accumulation of hydrogen peroxide $\left(\mathrm{H}_{2} \mathrm{O}_{2}\right)$ and superoxide radical $\left(\mathrm{O}_{2}^{-\cdot}\right)$ compared to wild-type (WT) plants under drought conditions. OsWRKY55 was expressed in various tissues and was induced by drought and abscisic acid (ABA) treatments. Through yeast two-hybrid assays, we found that OsWRKY55 interacted with four mitogen-activated protein kinases (MAPKs) that could be induced by drought, including OsMPK7, OsMPK9, OsMPK20-1, and OsMPK20-4. The activation effects of the four OsMPKs on OsWRKY55 transcriptional activity were demonstrated by a GAL4-dependent chimeric transactivation assay in rice protoplasts. Furthermore, OsWRKY55 was able to reduce plant height under normal conditions by decreasing the cell size. In addition, based on a dual luciferase reporter assay, OsWRKY55 was shown to bind to the promoter of OsAP2-39 through a yeast one-hybrid assay and positively regulate OsAP2-39 expression. These results suggest that OsWRKY55 plays a critical role in responses to drought stress and the regulation of plant height in rice, further providing valuable information for crop improvement.
\end{abstract}

Keywords: rice; transcription factor; OsWRKY55; drought response; plant growth; OsAP2-39

\section{Introduction}

As an adverse environmental factor, drought stress greatly constrains plant growth and productivity [1]. To withstand this stress, plants have evolved a complex series of actions that manipulate the expression levels of certain sets of genes. These actions include signal perception and transmission. Transcription factors (TFs) are involved and play a vital role in these signaling cascades [2,3]. In rice, which is often used as a monocot plant model, many TFs have been characterized using forward and/or reverse genetic approaches, including MYB, WRKY, Apetala2 (AP2)/ethylene-responsive factor (ERF), basic region-leucine zipper (bZIP), NAC (NAM, ATAF1/2, CUC1/2), and basic helix-loophelix (bHLH) [4-8].

Plant-specific WRKY TFs, one of the largest families of transcriptional regulators, are characterized by possessing a WRKY-DNA-binding domain [9]. Investigations of the overexpression or knockdown lines have shown that most WRKY TFs are involved in the plant immune response [10-13]. WRKY TFs also play crucial roles in various abiotic stress responses. The heterologous overexpression of either ZmWRKY106 or ZmWRKY40 
in Arabidopsis was found to confer drought tolerance [14,15], and the overexpression of GmWRKY12 in Arabidopsis was found to enhance tolerance to drought and salt. Similarly, GmWRKY54 was found to confer drought tolerance in soybean $[16,17]$, while the overexpression of OsWRKY76 improved tolerance to cold stress in rice [18]. However, the mechanisms by which WRKY TFs participate in drought stress responses in rice have not been fully elucidated. Plant mitogen-activated protein kinase (MAPK) cascades play important roles in different physiological responses, including biotic and abiotic stresses [19]. A few MAPK cascade genes have been identified to function in response to biotic and abiotic stresses through WRKY TFs $[20,21]$. OsWRKY30 is phosphorylated by MAP kinases, thereby enhancing drought tolerance in rice [22], and OsBWMK1 mediates defense responses by activating OsWRKY33 to regulate the expression of several pathogenesis-related genes [23]. OsWRKY53 interacts with OsMPK3/OsMPK6 and inhibits their activity [24].

The roles of several WRKY genes in plant growth and developmental processes, such as seed dormancy, germination, leaf senescence, and plant height, were also identified in previous studies. For example, in rice, the Oswrky 29 mutant displayed enhanced seed dormancy [25]. In Arabidopsis, a lack of WRKY41 reduced primary seed dormancy [26]. The heterologous overexpression of OsWRKY23 in Arabidopsis accelerated leaf senescence in darkness [27]. WRKY53 was found to act as a positive regulator of senescence, while WRKY70 negatively regulated leaf senescence in Arabidopsis [28,29]. Moreover, the enhanced expression of OsWRKY11.2 resulted in a semi-dwarf phenotype [30]. However, the functions of a number of WRKY TFs in rice remain to be elucidated.

Previously, overexpression of the WRKY family gene OsWRKY55 (LOC_Os03g20550, named OsWRKY55 by Xie et al. [31] and OsWRKY31 by Zhang et al. [32]) in rice was shown to enhance resistance to Magnaporthe grisea infection and induce the expression of defense-related genes, as well as affect root growth and auxin responses [32]. In this study, we characterized the other underlying roles of OsWRKY55 in rice. OsWRKY55 not only modulates rice height but is also involved in drought stress. The overexpression of OsWRKY55 in rice leads to a reduction in drought tolerance and plant height. Our findings reveal that OsWRKY55 plays crucial roles in the responses to drought stress and in the determination of plant height.

\section{Results}

\subsection{Overexpression of OsWRKY55 Reduces Drought Tolerance in Rice}

Previously, we constructed transgenic rice plants overexpressing different TFs to explore the roles of TFs in response to environmental stresses [33]. We identified two transgenic lines overexpressing OsWRKY55 by surveying the phenotypes of transgenic lines overexpressing the WRKY gene under drought stress treatment. Using quantitative reverse transcription PCR (qRT-PCR), we verified that OsWRKY55 was significantly upregulated in two independent transgenic lines, OE-1 and OE-2, and the expression level of the endogenous OsWRKY55 gene in transgenic rice plants was similar to that in wild type (WT) (Figure 1A,B and Figure S1).

The seedlings overexpressing OsWRKY55 (OsWRKY55-OE) and wild-type (WT) plants were phenotypically indistinguishable under normal conditions. However, leaf wilt was much more severe in the transgenic seedlings than in the WT seedlings under drought stress (Figure 1C). After 7 days of recovery (re-watering), the survival rates of the WT seedlings $(\approx 65-80 \%)$ were significantly greater than those of the transgenic OsWRKY55-OE seedlings $(\approx 45-53 \%$; Figure $1 \mathrm{D})$, suggesting that OsWRKY55 negatively regulates drought resistance in rice. This hypothesis was tested by a detached leaf water loss assay. The transgenic OsWRKY55-OE lost water faster than the WT (Figure 1E). Since abiotic stress may lead to the accumulation of excessive reactive oxygen species (ROS) in plants, resulting in cell damage, we measured ROS accumulation using histochemical assays. Ultimately, 3, 3'-diaminobenzidine (DAB) staining indicated that under drought conditions, more hydrogen peroxide $\left(\mathrm{H}_{2} \mathrm{O}_{2}\right)$ was accumulated in the leaves of the OsWRKY55-OE plants than in those of the WT plants, as evidenced by the abundance of brown spots in the 
OsWRKY55-OE leaves; there were no visible differences between OsWRKY55-OE and WT leaves under normal conditions (Figure 1F). Although both WT and OsWRKY55-OE plants showed evidence of superoxide radical $\left(\mathrm{O}_{2}^{-\cdot}\right)$ accumulation under drought conditions (as indicated by blue dots on the leaves after nitro-blue tetrazolium (NBT) staining), fewer, lighter dots were also observed on the WT leaves; there were no visible differences between the OsWRKY55-OE and WT leaves under normal conditions (Figure 1G). These results indicate that the overexpression of OsWRKY55 may increase ROS production, thereby decreasing drought resistance.

A
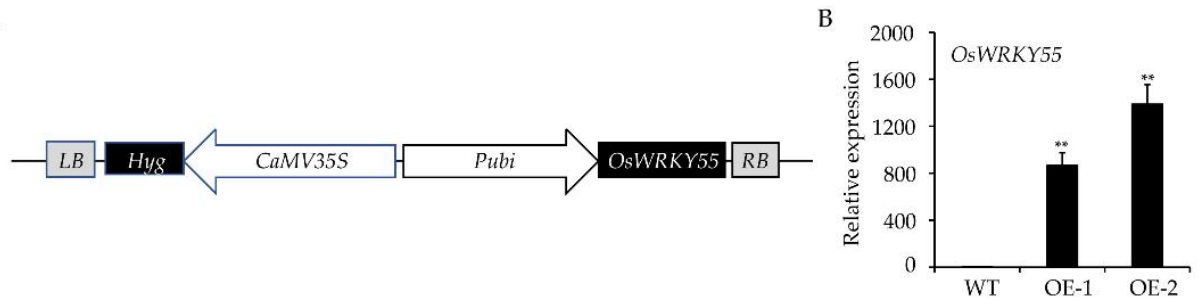

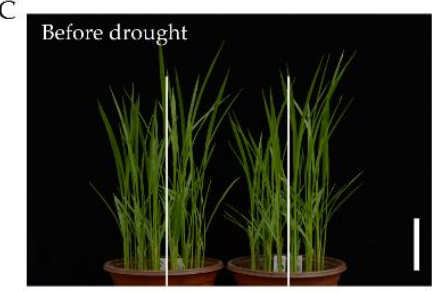

OE-1 WT OE-2 WT

$\mathrm{E}$

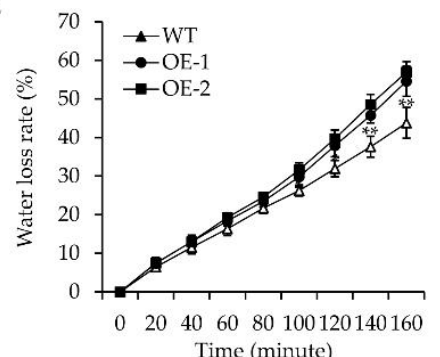

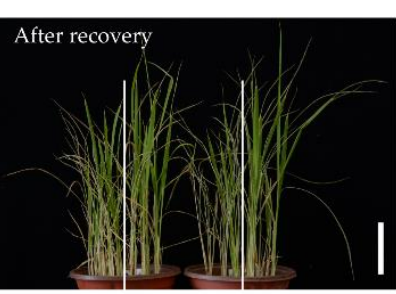
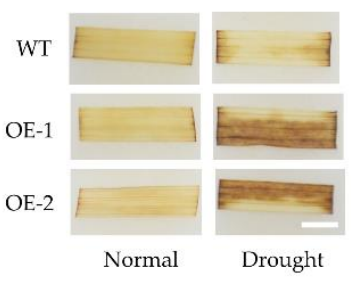

$$
\text { D }
$$

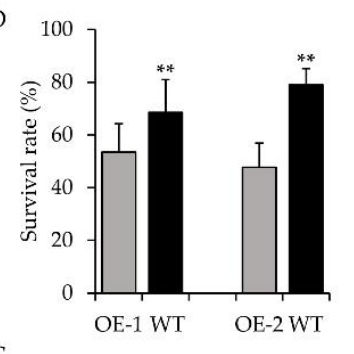

G

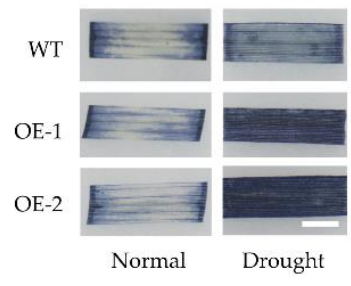

Figure 1. Overexpression of OsWRKY 55 increases the sensitivity of rice seedlings to drought stress. (A) Diagrams of the overexpression plasmid of OsWRKY55. (B) The OsWRKY55 mRNA level in each genotype was analyzed by quantitative reverse transcription PCR (qRT-PCR). The rice OsActin gene was used as the internal control. (C) Appearance of wild-type (WT) and transgenic seedlings (OE-1 and OE-2) before drought stress and after 7 days of recovery (rewatering). Scale bars $=5 \mathrm{~cm}$. (D) Survival rates of WT and transgenic seedlings after recovery for 7 days. (E) Water loss assay of WT, OE-1, and OE-2. (F) 3, 3'-diaminobenzidine (DAB) staining showing the level of hydrogen peroxide $\left(\mathrm{H}_{2} \mathrm{O}_{2}\right)$ in the leaves of WT and transgenic plants under normal and drought-stress conditions. Scale bars $=0.5 \mathrm{~cm}$. $(\mathrm{G})$ Nitro-blue tetrazolium (NBT) staining showing the level of superoxide radical $\left(\mathrm{O}_{2}^{-\cdot}\right)$ in the leaves of WT and transgenic plants under normal and drought-stress conditions. Scale bars $=0.5 \mathrm{~cm}$. In all graphs, values shown are means $\pm \mathrm{SD}(\mathrm{n}=3),{ }^{* *} p<0.01$ (Student's $t$-test).

\subsection{Expression Pattern of OsWRKY55}

To further explore the function of OsWRKY55 in rice, we first detected the expression profile of OsWRKY55 under drought stress and abscisic acid (ABA) treatment. OsWRKY55 was strongly up-regulated after drought stress. OsWRKY55 expression peaked in the leaves after $6 \mathrm{~h}$ of drought (Figure 2A) and in the roots after $0.5 \mathrm{~h}$ of drought (Figure 2B). QRT-PCR analyses showed that the induced expression patterns of OsWRKY55 in leaves and roots under ABA treatment were similar to those after drought stress (Figure 2C,D). In addition, we also assessed the tempo-spatial expression profile of this gene via qRT-PCR. OsWRKY55 was constitutively expressed in all tissues analyzed, with relatively higher expression 
levels in roots and flag leaves and relatively lower expression levels in young leaves and stems (Figure 2E). As the preliminary results suggested that OsWRKY55 is associated with drought response, we next analyzed the promoter sequence of the OsWRKY55 gene using the PlantCARE database (http:/ / sphinx.rug.ac.be:8080/PlantCARE/, accessed on 15 September, 2020). Analysis of the OsWRKY55 gene promoter sequence identified many putative stress response-related cis-elements, including the MYB recognition site (5 hits), ABA-responsive element (ABRE) (5 hits), W-box (3 hits), and MYC recognition site (2 hits) (Figure 2F).
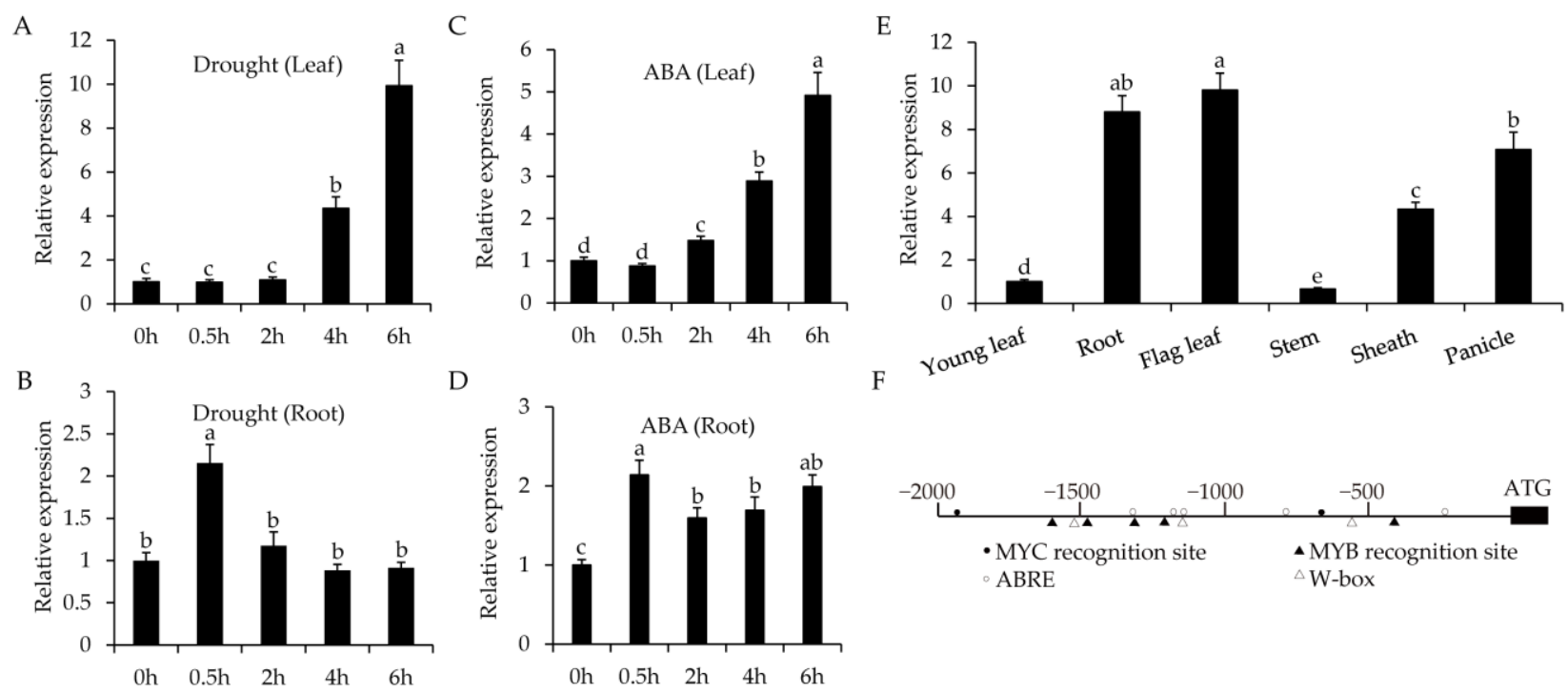

Figure 2. Expression pattern analysis of OsWRKY55. (A,B) Relative expression of OsWRKY55 in leaves (A) and roots (B) after drought treatment (dehydration). (C,D) Relative expression of OsWRKY55 in leaves (C) and roots (D) after treatment with $100 \mu \mathrm{M}$ of the phytohormone abscisic acid (ABA). (E) Expression profile of OsWRKY55 in various rice tissues of the wild-type (WT). (F) Distribution of stress-response-related cis-elements in the $2 \mathrm{~kb}$ promoter region of OsWRKY55. In all graphs, values shown are means $\pm \mathrm{SD}(\mathrm{n}=3)$. The rice OsActin gene was used as the internal control. Different letters above the bars indicate significant differences $(p<0.05)$.

\subsection{OsWRKY55 Interacts with Several OsMPKs}

To determine whether OsWRKY55 interacts with OsMPKs, the full-length coding sequences (CDSs) of 15 OsMPKs [34] were fused to separate pGADT7 as prey, and pGBKT7OsWRKY55 was used as bait in the yeast two-hybrid assays. The results showed that all transformants grew well on the SD/-Trp-Leu-His plates containing $0 \mathrm{mM}$ 3-amino1, 2, 4-triazole (3-AT) (Figure 3A and Figure S2) due to the auto-transcriptional activity of OsWRKY55 in yeast [32]. Only three transformants (carrying BD-OsWRKY55/ADOsMPK20-1, BD-OsWRKY55/AD-OsMPK20-4, or BD-OsWRKY55/AD-OsMPK9) grew well on the SD/-Trp-Leu-His plates containing 0.2 and $0.4 \mathrm{mM}$ 3-AT (Figure 3A and Figure S2). However, the growth of cells carrying BD-OsWRKY55/AD-OsMPK7 was reduced on the $0.2 \mathrm{mM}$ 3-AT plate and inhibited on the $0.4 \mathrm{mM}$ 3-AT plate (Figure 3A). Negative control yeast cells carrying BD-OsWRKY55/AD did not grow well on SD/Trp-Leu-His plates containing 0.2 and $0.4 \mathrm{mM} 3$-AT. These results indicate that, in yeast, OsWRKY55 interacts strongly with OsMPK20-1, OsMPK20-4, and OsMPK9 but weakly with OsMPK7 (Figure 3A). 
A
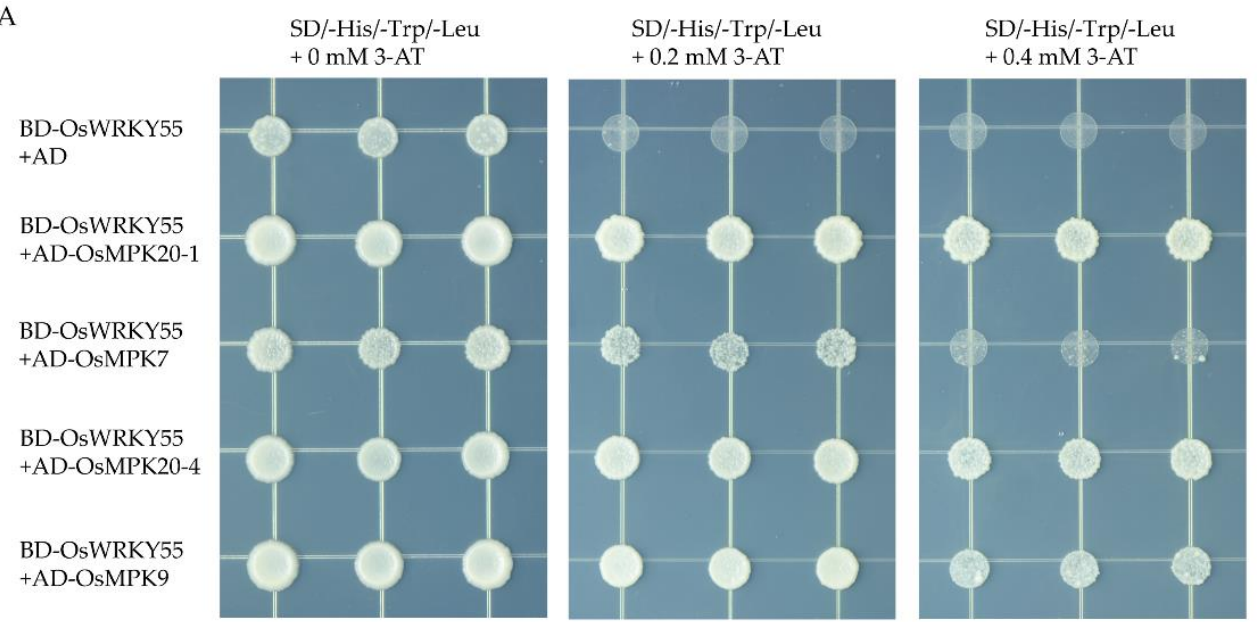

B

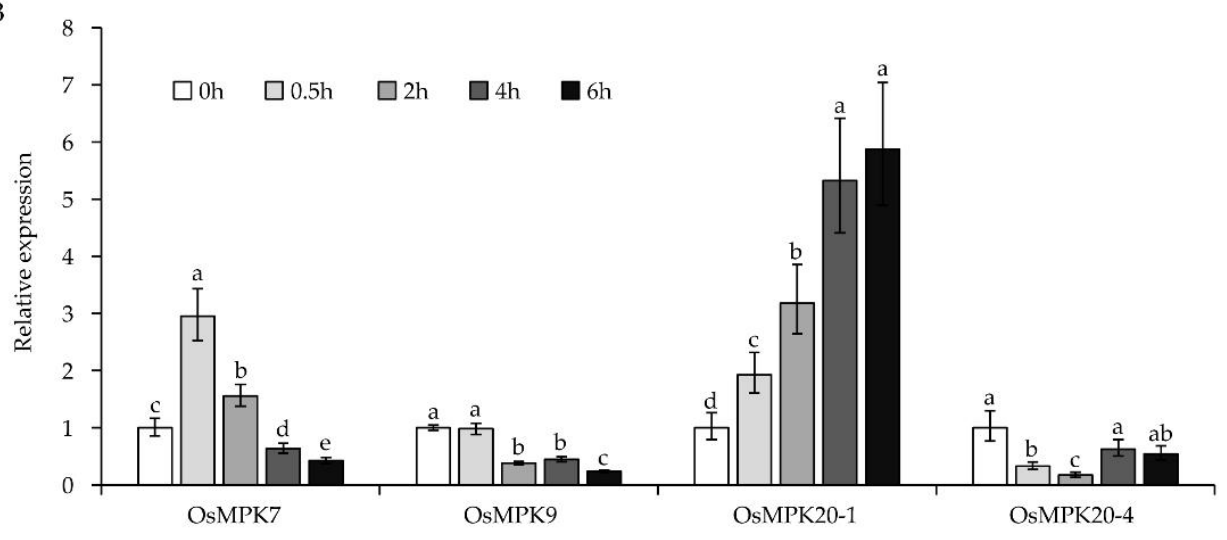

C

Effector

\begin{tabular}{|c|c|c|c|}
\hline & p35s & GAL4DB & - GAL4DB \\
\hline & p35s & GAL4DB & OsWRKY55 GAL4DB-OsWRK \\
\hline & p35s & OsMPK & K7 — OsMPK7 \\
\hline & p35s & OsMPK & K9 \\
\hline & p35s & OsMPK2 & $20-1$ - OsMPK20-1 \\
\hline & p35s & OsMPK2 & $20-4$ - OsMPK20-4 \\
\hline \multicolumn{4}{|c|}{ Reporter } \\
\hline & \multicolumn{2}{|c|}{ 5×GAL4 TATA } & FLUC- \\
\hline \multicolumn{4}{|c|}{ Internal control } \\
\hline & p35S & RLUC & - \\
\hline
\end{tabular}

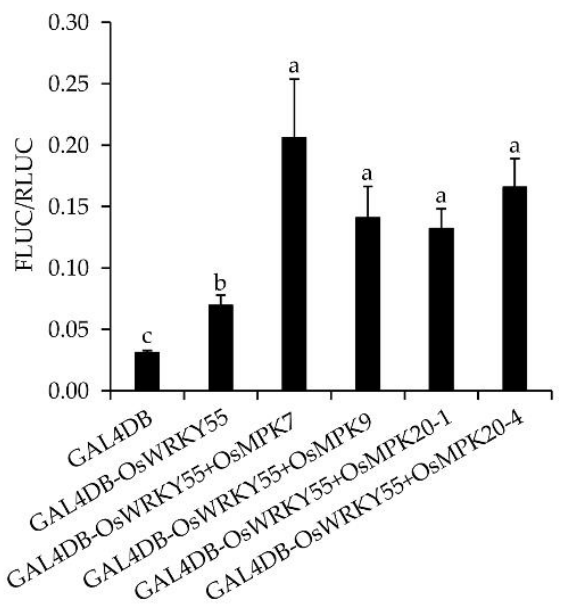

Figure 3. OsMPKs interact with OsWRKY55 and increase its transcription activity. (A) The interactions between OsWRKY55 and four OsMPKs in the yeast two-hybrid assays. Growth phenotypes of yeast cells on selective media plates (SD/-Trp-Leu-His) containing 0, 0.2, or 0.4 mM 3-amino-1, 2, 4-triazole (3-AT). (B) Relative expressions of OsMPK7, OsMPK9, OsMPK20-1, and OsMPK20-4 after drought treatment (dehydration). (C) GAL4-dependent chimeric transactivation assay of OsWRKY55 in rice protoplasts. A schematic illustration of the effector and reporter vectors is shown on the left. FLUC, firefly luciferase; RLUC, renilla luciferase. The right part is the transcription activities by co-transformation of different effector vector(s) with the reporter vector and internal control. Values shown are means $\pm \mathrm{SD}(\mathrm{n}=3)$. The rice OsActin gene was used as the internal control. Different letters above the bars indicate significant differences $(p<0.05)$. 
To examine the possible involvement of these four OsMPKs in the drought stress response, we assessed the expression patterns of OsMPKs under drought stress via qRTPCR. We found that the expression level of OsMPK7 was increased within $0.5 \mathrm{~h}$ and that OsMPK9 expression was down-regulated rapidly after $2 \mathrm{~h}$ of drought treatment. Under drought stress, the expression of OsMPK20-1 was significantly induced, and OsMPK20-4 was suppressed after $0.5 \mathrm{~h}$, but the expression resumed slightly after $4 \mathrm{~h}$ (Figure 3B).

To further explore whether the four OsMPKs affect the activity of OsWRKY55, a GAL4dependent chimeric transactivation assay in rice protoplasts was performed. The results illustrated the activation effects of the four OsMPKs on OsWRKY55 transcriptional activity (Figure 3C), which also suggested that the interactions between OsWRKY55 and OsMPKs occurred in vivo.

\subsection{Overexpression of OsWRKY55 Reduces Plant Height}

OsWRKY55 was not only associated with the drought stress response but was also observed to affect plant growth. The transgenic OsWRKY55-OE lines grew more slowly than the WT plants (Figure 4A). At maturity, the two OsWRKY55-OE lines were significantly shorter than those of the WT plants-OE1 and OE2 were 25.4\% and 27.8\% shorter than the WT, respectively (Figure 4B). All four internodes of each OsWRKY55-OE line were significantly shorter than the corresponding WT internodes (Figure 4C,D). A microscopic examination of the longitudinal sections of the uppermost internodes of the main culms of the WT and OsWRKY55-OE (OE-1) plants showed that there were noticeable differences in cell shape between the WT and transgenic plants. Indeed, there were significant differences in both cell length and cell width between the WT and OsWRKY55-OE (OE-1) plants (Figure 5A,B). Thus, our results indicate that OsWRKY55 affects plant height by negatively regulating cell expansion.
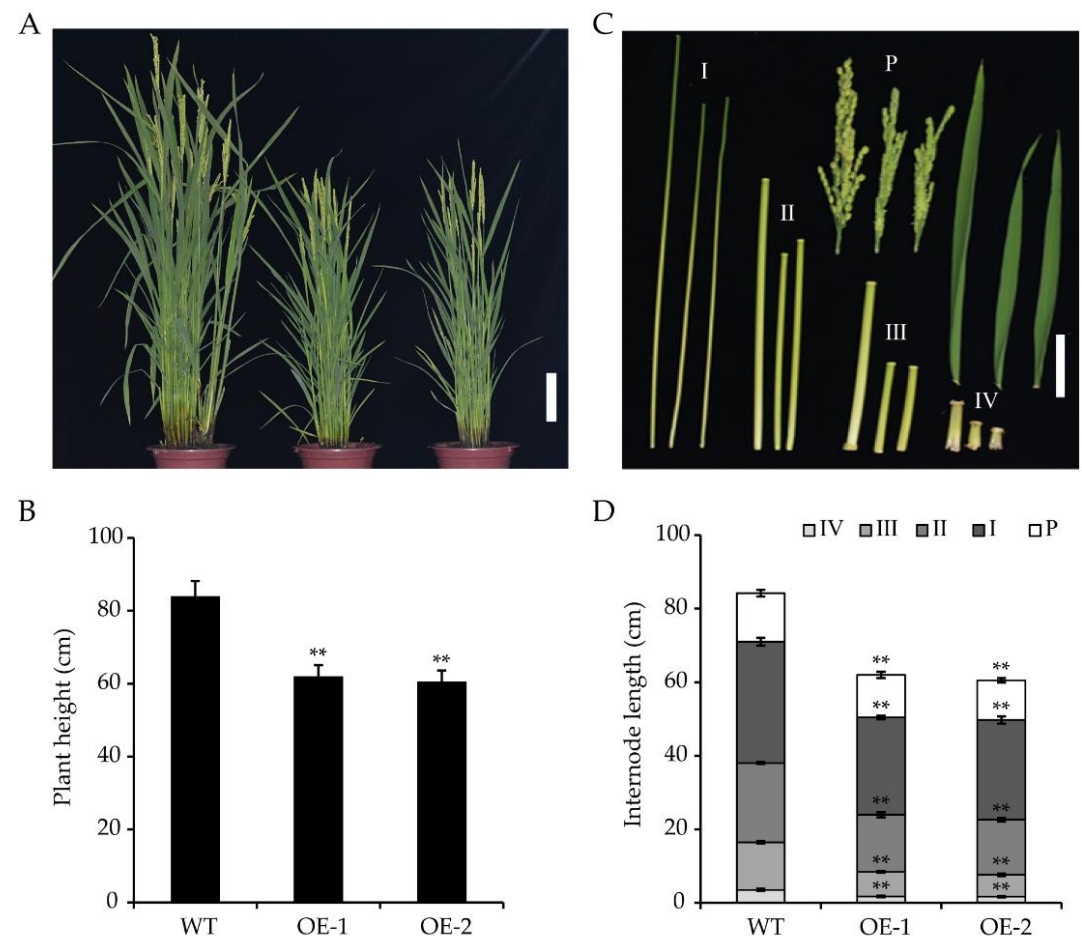

Figure 4. Overexpression of OsWRKY55 reduces plant height. (A) Phenotypes of wild-type (WT) plants and transgenic lines overexpressing OsWRKY55 (OE-1 and OE-2). Scale bar $=10 \mathrm{~cm}$. (B) Heights of mature WT and OE plants. (C) Panicles (P) and internodes (I-IV) of mature WT and OsWRKY55-OE plants. Scale bar $=5 \mathrm{~cm}$. (D) Lengths of panicles $(\mathrm{P})$ and internodes (I-IV) in mature WT and OsWRKY55-OE plants. In all graphs, values shown are means $\pm \operatorname{SD}(\mathrm{n} \geq 15),{ }^{* *} p<0.01$ (Student's $t$-test). 
A

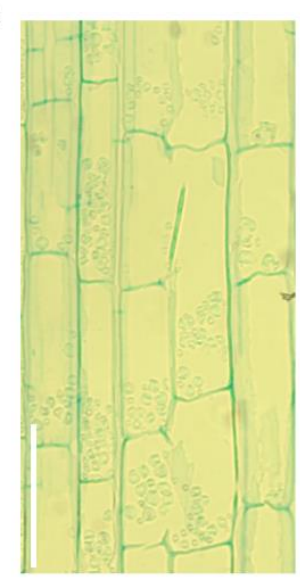

WT

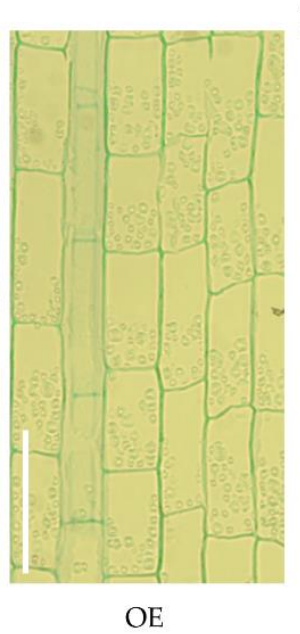

B
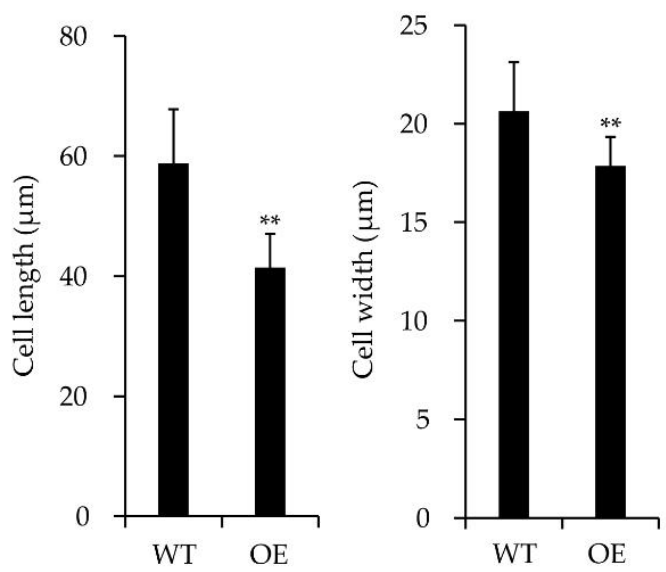

Figure 5. Overexpression of OsWRKY55 decreases cell size. (A) Longitudinal sections of the uppermost internodes of the main culms of the wild-type (WT) plants and transgenic lines overexpressing OsWRKY55 (OsWRKY55-OE-1). Scale bars $=50 \mu \mathrm{m}$. (B) Cell size comparison of the uppermost internodes of the main culms in WT and OsWRKY55-OE (OE-1) lines. Values shown are means \pm SD $(\mathrm{n}=3),{ }^{* *} p<0.01$ (Student's $t$-test).

\subsection{OsAP2-39 Is Directly Regulated by OsWRKY55}

To elucidate the molecular function of OsWRKY55, we identified the target genes of OsWRKY55 through the genome-wide expression profile changes in WT and OsWRKY55$\mathrm{OE}$ lines via an RNA-sequencing (RNA-seq) assay under normal growth conditions at the four-leaf stage. The result revealed that about 162 genes in overexpressing OsWRKY 55 rice experienced two-fold changes compared to the genes in the WT rice. Among these genes, 98 were up-regulated in the OE lines. Although the predicted functions of the up-regulated genes were extremely diverse, there were many stress-related genes, such as OsAP2-39 (LOC_Os04g52090), OsERF48/OsDRAP1 (LOC_Os08g31580), OsFbx352 (LOC_Os10g03850), OsRAV2 (LOC_Os01g04800), OsMYB48 (LOC_Os01g74410), and Zinc finger protein (LOC_Os03g55540, LOC_Os03g60570, LOC_Os01g61420, LOC_Os02g45710) (Table S2). Therefore, these genes might be direct or indirect targets of OsWRKY55. As OsAP2-39, the APETALA-2-like transcription factor is known to negatively regulate plant growth and drought tolerance [35], we further investigated an experimentally verified gene, OsAP2-39 (Figure 6A). The up-regulation of OsAP2-39 in transgenic lines overexpressing OsWRKY 55 was verified by qRT-PCR (Figure 6B).

To further identify whether OsAP2-39 is the target gene of OsWRKY55, a yeast onehybrid assay and a dual luciferase reporter assay were performed. The $\beta$-galactosidase activity level in the yeast cells co-transformed with pLacZi-Pro and pGAD424-OsWRKY55 was significantly greater than that of the control ( $\approx$ 10 -fold increase in activity; Figure $6 \mathrm{C}$ ), indicating that OsWRKY55 binds to the OsAP2-39 promoter in yeast. Furthermore, the dual luciferase reporter assay showed that the FLUC/RLUC ratio, which reflects transcriptional activity, was significantly up-regulated in rice protoplasts harboring the effector vector pAN580-OsWRKY55 compared to that in protoplasts carrying the vector pAN580 (Figure 6D). These results suggest that OsAP2-39 is a target gene of OsWRKY55. 

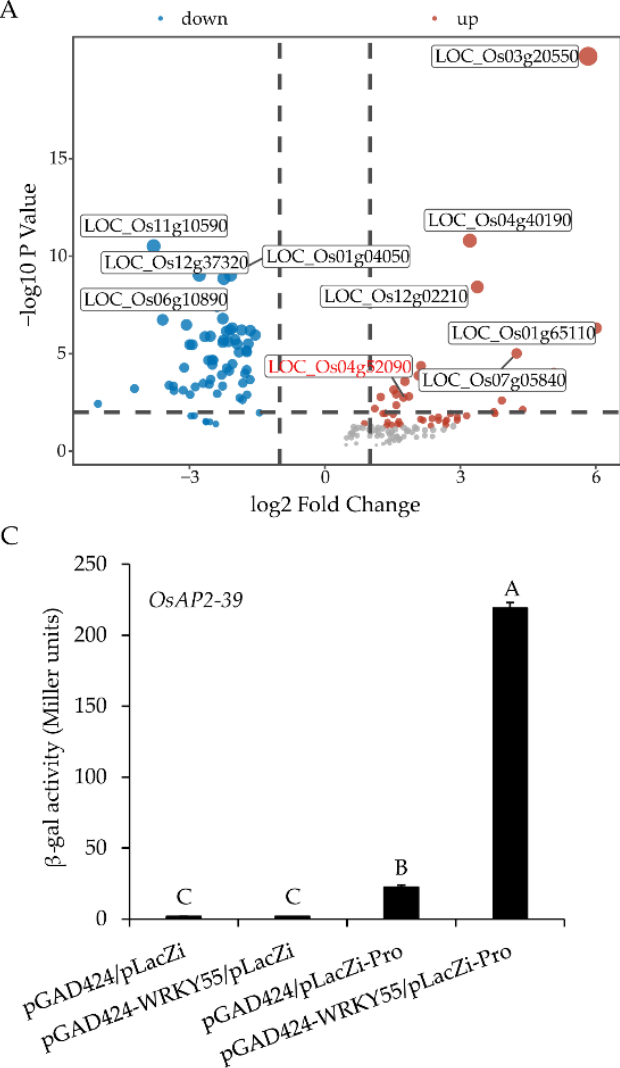

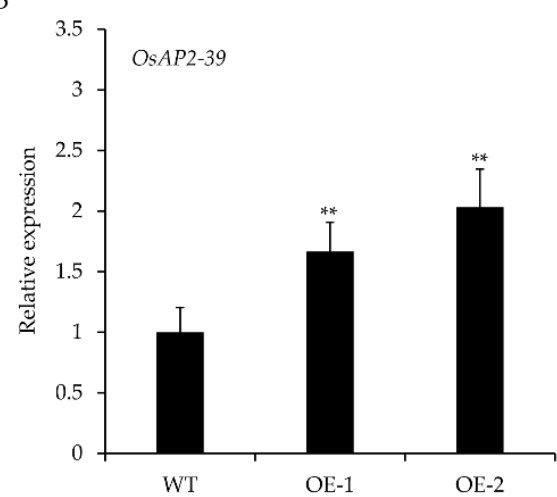

D

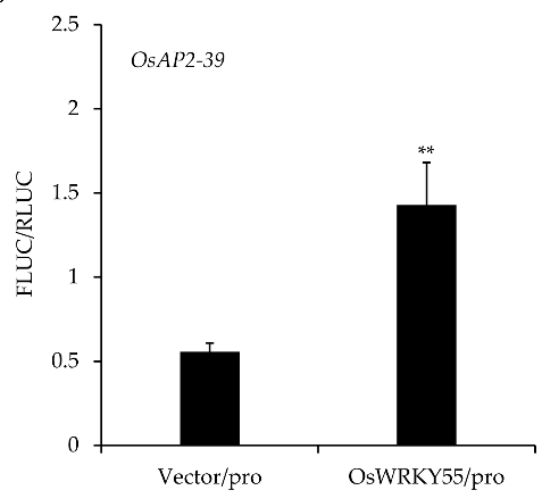

Figure 6. OsWRKY55 positively regulates the expression of OsAP2-39. (A) Identification of differentially expressed genes (DEGs) in transgenic lines overexpressing OsWRKY55 compared to the wild type (WT) under normal conditions via RNA-sequencing (RNA-seq) analysis. The blue and red spots represent down-regulated and up-regulated genes, respectively. The sizes of the spots indicate the $-\log _{10}$ FDR. (B) Relative expression of the OsAP2-39 gene in WT plants and transgenic lines overexpressing OsWRKY55 (OE-1 and OE-2). The rice OsActin gene was used as the internal control. Values shown are means $\pm \mathrm{SD}(\mathrm{n}=3),{ }^{* *} p<0.01$ (Student's $t$-test). (C) $\beta$-galactosidase activity levels in co-transformed yeast cells. Values shown are means $\pm S D(n=3)$. Different uppercase letters above the bars indicate significant differences (least significant differences, $p<0.01$ ). (D) Transcriptional activity of OsAP2-39 in rice protoplasts harboring the effector vector pAN580-OsWRKY55 compared to that in protoplasts carrying the empty vector. The transient expression of FLUC driven by the promoter of OsAP2-39 was normalized to the internal control reporter (RLUC). Values shown are means $\pm \mathrm{SD}(\mathrm{n}=3),{ }^{* *} p<0.01$ (Student's $t$-test).

\section{Discussion}

Rice is negatively affected by various abiotic and biotic stresses, resulting in serious yield losses worldwide. Previous studies have identified that many WRKY TFs participate in the rice response to biotic stresses [36]. However, to date, our understanding of the molecular mechanisms by which WRKY TFs in rice are involved in abiotic stresses, especially drought stress, remains largely unknown. Furthermore, the roles of WRKY TFs in the regulation of growth and development in rice have not yet been studied extensively. A prior study showed that OsWRKY55 enhanced resistance against infection with $M$. grisea and affected root growth and auxin response [32]. In the present work, we focused on the response of OsWRKY55 to drought stress and the regulation of plant height. Under drought treatment, by withholding the water supply, OsWRKY55-OE plants became more sensitive to drought stress than WT plants (Figure 1C,D), which is likely because the OsWRKY55-OE plants lost water at a faster rate (Figure 1E) and had fewer, shorter lateral roots than the WT plants [32]. Furthermore, the OsWRKY55-OE plants accumulated more $\mathrm{H}_{2} \mathrm{O}_{2}$ and $\mathrm{O}_{2}{ }^{-}$ under drought conditions (Figure 1F,G). The associated increase in oxidative damage might 
also explain the increased susceptibility of the OsWRKY55-OE transgenic lines to drought. We also found that the overexpression of OsWRKY 55 reduced plant growth by decreasing cell size under normal conditions: OsWRKY55-OE plants were shorter than WT plants, with smaller, thinner cells in the internodes (Figures 4 and 5). A previous study revealed that OsWRKY55 is an auxin-inducible gene and that the overexpression of OsWRKY55 enhances auxin-related phenotypes in rice [32]. Auxin regulates a variety of processes during plant development [37]. However, it remains unclear whether the involvement of OsWRKY55 in auxin signaling leads to the dwarfism observed in the OsWRKY55-OE plants.

MAPK cascades in plants are involved in various physiological functions. WRKY TFs and MAPK cascades have been shown to modulate plant stress response [19,38-42]. Under biotic and abiotic stress, many WRKY TFs are activated by MAPKs. For example, OsMPK6 phosphorylates and activates OsWRKY45. However, under cold and salinity stress, a tyrosine protein phosphatase dephosphorylates and inactivates OsMPK6, which reduces OsWRKY45 defense [43]. OsMPK7and OsMPK20-4 interact with OsWRKY30, and OsMPK7 further phosphorylates OsWRKY30, which are both crucial processes for OsWRKY30 to confer drought tolerance in rice [22]. In this study, we demonstrated that OsWRKY55 interacts with four different MAPKs in yeast, including OsMPK7, OsMPK9, OsMPK20-1, and OsMPK20-4 (Figure 3A), and that the four OsMPKs can activate the transcriptional activity of OsWRKY55. The qRT-PCR results showed that these four OsMPKs may be related to drought stress response (Figure $3 \mathrm{~B}$ ). These results suggest that the decreased drought tolerance associated with OsWRKY55 may, to a large extent, be due to the interactions between OsWRKY55 and these four OsMPKs. OsWRKY30 and OsWRKY55 can be activated by OsMPK7, but they confer different drought tolerance levels in rice, which indicates that the regulatory network of responses to drought stress in rice is complex. Overexpression of the OsWRKY55 gene was found to enhance resistance against infection with $M$. grisea [32]. It was suggested that OsWRKY55 plays an opposite role in biotic and abiotic stress, which may be due to the regulation of different downstream genes by OsWRKY55.

$\mathrm{AP2}$ /ERF proteins play important roles in plant responses to biotic and abiotic stress, as well as in the growth and development in rice. For example, OsAP2-39, a member of the APETALA2 (AP2) family, affects dehydration tolerance and plant height in rice by controlling the ABA/gibberellin (GA) balance [35]. However, overexpressing OsAP2-39 lines display different response results depending on the different ways in which they are grown under drought stress. When grown in the same pot, transgenic OsAP2-39 plants exhibited lower dehydration tolerance than WT plants, which is likely due to smaller root systems of overexpressing OsAP2-39 lines, which are similar to those of the drought-sensitive phenotype of OsWRKY55-OE plants. When grown in separate pots, the responses were reversed, which was likely because the transpiration rates of the WT plants were greater. The definitive conclusion, that overexpressing OsAP2-39 lines had lower dehydration tolerance than the WT, was reached via an excised leaf water loss assay. It was reported that an ERF protein, OsDERF1, activates OsERF3 and OsAP2-39 to negatively modulate ethylene synthesis and drought tolerance in rice [44]. The present study revealed that OsWRKY55 can bind to the promoter of OsAP2-39 to up-regulate the expression of OsAP2-39 (Figure 6), suggesting that the OsWRKY55 and OsAP2-39 transcriptional cascade negatively modulates drought response. OsDERF1 and OsWRKY55 both regulate drought tolerance by activating OsAP2-39 in rice, but whether they function in the same pathway remains to be elucidated.

In conclusion, the overexpression of OsWRKY 55 could reduce drought resistance and plant height in rice. Four OsMPKs interacted with OsWRKY55 and activated OsWRKY55. Moreover, OsWRKY55 was able to directly regulate OsAP2-39. This work demonstrates the novel function of OsWRKY55 in rice. 


\section{Materials and Methods}

\subsection{Plant Materials and Growth Conditions}

Wild-type rice seedlings (WT, Oryza sativa japonica cv Kitaake) and two homozygous $\mathrm{T} 3$ transgenic rice lines overexpressing OsWRKY 55 (OE-1 and OE-2) were used in these experiments. To generate OsWRKY55-OE overexpression lines, the full-length coding sequence (CDS) of OsWRKY55 was amplified from rice seedlings of Kitaake and cloned into the vector pCUbi1390 under the control of the maize ubiquitin (Ubi) promoter. The constructed vector $p U b i$ :OsWRKY55 was introduced into rice (Kitaake) using Agrobacterium tumefaciens (EHA105)-mediated transformation. The homozygous lines were selected via hygromycin resistance evaluation. Rice overexpressing OsWRKY55 is denoted as OE, and the different transgenic lines are indicated with numbers.

Seeds were submerged in water at $28^{\circ} \mathrm{C}$ for 3 days. Then, the uniformly germinated seeds were transplanted into containers filled with Yoshida's culture solution and boxes filled with soil from a paddy field in a growth chamber $\left(28^{\circ} \mathrm{C}, 80 \%\right.$ relative humidity and a 14/10 $\mathrm{h}$ day/night photoperiod), respectively.

\subsection{Stress Treatments}

Phenotypic analysis of drought stress was conducted at the seedling stage. When OsWRKY55-OE lines and WT plants growing in the soil reached the four-leaf stage, irrigation was withheld for 7 days, and then the seedlings were allowed to recover for 7 days. Seedlings that did not grow were considered not to have survived. The survival rates were calculated as the percentage of seedlings that survived among the total treated seedlings. WT and transgenic plants were grown to maturity in the paddy field under normal conditions. At the booting stage, we measured lengths of the panicles and internodes and plant heights.

To determine the OsWRKY55 gene expression profile under stress conditions, fourleaf-stage WT rice seedlings growing in the hydroponic culture medium were subjected to drought stress (by removing the water supply) and phytohormone ABA treatment (100 $\mathrm{MM}$ ABA added to the culture medium) before being sampled at $0,0.5,2,4$, and $6 \mathrm{~h}$. To characterize the spatiotemporal expression of OsWRKY 55 , young leaves and roots were collected from the WT seedlings at the four-leaf stage in the soil under normal conditions, and samples of the flag leaves, stems, sheaths, and panicles were collected from WT plants at the booting stage described above.

\subsection{Water Loss Assay}

The OsWRKY55-OE and WT plants were grown in pots under normal growth conditions for 4 weeks. The leaves were detached from the plants and weighed immediately as the initial weight. The samples were exposed to air at room temperature and then weighed at the same time intervals $(20 \mathrm{~min})$ after being cut down. The rate of water loss was calculated based on the initial weights of the samples. Three replicates were made for each line.

\subsection{Quantification of Gene Expression}

Total RNA was extracted from each sample using the RNAprep Pure Plant Kit (Tiangen Biotech). First-strand cDNA was synthesized with $2 \mu \mathrm{g}$ of total RNA using a RevertAid First Strand cDNA Synthesis Kit (Thermo Scientific Fermentas). Quantitative RT-PCR reaction was performed using a Mx3005P instrument (Stratagene) with the SYBR Green Real-time PCR Master Mix (Toyobo) according to the following conditions: 1 cycle of $2 \mathrm{~min}$ at $95^{\circ} \mathrm{C}, 40$ cycles of $15 \mathrm{~s}$ at $95^{\circ} \mathrm{C}$, and $30 \mathrm{~s}$ at $60{ }^{\circ} \mathrm{C}$, followed by 1 cycle of $60 \mathrm{~s}$ at $95^{\circ} \mathrm{C}$, $30 \mathrm{~s}$ at $55^{\circ} \mathrm{C}$, and $30 \mathrm{~s}$ at $95^{\circ} \mathrm{C}$ for the melting curve analysis. The rice OsActin gene and Ubiquitin5 gene (Supplemental Figure S3) were used as the internal controls against which to calculate relative expression. Relative expression levels were calculated using the $2^{-\Delta \Delta C t}$ method [45]. The experiment was conducted using three biological replicates. The primers used are listed in Table S1. 


\subsection{RNA Sequencing}

Four-leaf-stage WT and the OsWRKY55-OE plants under normal conditions were sampled for RNA sequencing (RNA-seq). Total RNA of the leaves was extracted using the RNAprep Pure Plant Kit (Tiangen Biotech). cDNA library construction and next-generation sequencing were performed by Biomarker Bio-Tech Company (Beijing, China) on an Illumina Hiseq2500 platform $(2 \times 101 \mathrm{bp})$. Three biological replicates were performed. The raw reads were filtered using the FASTQ_Quality_Filter tool from the FASTX-Toolkit (http:/ / hannonlab.cshl.edu/fastx_toolkit, accessed on 11 May, 2020). Next, the clean reads were mapped to the rice reference genome (MSU, Rice Genome Annotation Release 7) using Hisat2, a spliced read mapper for RNA-seq (http:/ /ccb.jhu.edu/software/hisat2 /index.shtml, accessed on 11 May, 2020). Gene expression profiles were analyzed using Stringtie (Transcript assembly for RNA-seq), and transcript abundance was measured using the Fragment Per Kilobase of transcript sequence per Millions base pairs Sequenced (FPKM) method. Differentially expressed genes (DEGs) were identified using the DESeq package in $R$. The volcano map of DEGs was made using R package ggplot2.

\subsection{Yeast Two-Hybrid Assay}

To identify the interactions of OsWRKY55 and OsMPKs in yeast, a yeast two-hybrid assay was carried out according to the manufacturer's instructions (Clontech). The fulllength coding DNA sequence (CDS) of OsWRKY55 was fused to the pGBKT7 vector (BDOsWRKY55) as bait, and the full-length CDSs of the OsMPKs were cloned into separate pGADT7 vectors as prey. The bait vector was co-transformed with each prey vector into the Y2H Gold yeast strain and cultured on SD/-Trp-Leu plates at $30^{\circ} \mathrm{C}$ for $2-3$ days. Positive transformants were then cultivated on SD/-Trp-Leu-His plates containing $0,0.2$, or $0.4 \mathrm{mM}$ 3-amino-1, 2, 4-triazole (3-AT) at $30^{\circ} \mathrm{C}$ for 3 days.

\subsection{Yeast One-Hybrid Assay}

For the yeast one-hybrid assay, the promoter $(\approx 1500 \mathrm{bp})$ of OsAP2-39 was cloned into the reporter vector pLacZi (pLacZi-Pro). The full-length CDS of OsWRKY55 was amplified and fused to the vector pGAD424 (Clontech), which encodes the activation domain of the yeast GAL4 transcriptional activator. Then, the reporter vector pLacZi-Pro was digested using a restriction enzyme (either $\mathrm{NcoI}$ or ApaI) and co-transformed with the fusion protein into the yeast strain YM4271 (Clontech). The transformants were cultured on SD/-Leu-Ura plates at $30^{\circ} \mathrm{C}$ for 3 days. Putative positive clones were identified using DNA sequencing. Finally, $\beta$-galactosidase liquid assays were performed to quantify the DNA-protein interactions, following the instructions of the manufacturer (Clontech).

\subsection{GAL4-Dependent Chimeric Transactivation Assay}

The assay was performed in rice protoplasts, as described previously [46]. The OsWRKY 55 coding region was cloned into the effector vector with the GAL4 DNA-binding domain, while the full-length coding regions of OsMPK7, OsMPK9, OsMPK20-1, and OsMPK20-4 were cloned into the effector plasmid without the GAL4 DNA-binding domain. GAL4DB was used as a negative effector. The reporter was a plasmid containing the firefly luciferase (FLUC) gene. A plasmid harboring the Renilla LUC (RLUC) gene under control of the CaMV 35S promoter was used as an internal control. The effector plasmids were co-transformed with the reporter plasmid and internal control into rice protoplasts. After incubation at $28^{\circ} \mathrm{C}$ for $12-16 \mathrm{~h}$, the protoplasts were collected, and the results were reported as the ratios between the activity of FLUC and RLUC using a luciferase activity assay (Promega).

\subsection{Dual Luciferase Reporter Assay}

For the dual luciferase reporter assay, the OsAP2-39 promoter ( 1500 bp) was inserted into the vector pGreen II 0800 (which contained a firefly luciferase (FLUC) reporter gene and an internal control Renilla luciferase (RLUC) driven by the CaMV $35 \mathrm{~S}$ promoter) to 
generate the reporter vector. The CDS of OsWRKY55 was inserted into the pAN580 vector to generate the effector vector. Then, the reporter vector was co-transformed into rice protoplasts via PEG-mediated transformation [47] with either the empty pAN580 vector (as the negative control) or the effector vector (pAN580-OsWRKY55). After 12-16 h of culture at $28{ }^{\circ} \mathrm{C}$, the FLUC and RLUC activity levels in the rice protoplasts were measured using a Dual-Luciferase Reporter Assay System (E1910, Promega). The transient expression of LUC, driven by the promoter of OsAP2-39, was normalized to the expression of the internal control reporter (RLUC).

\subsection{Histochemical Analysis}

For 3, $3^{\prime}$-diaminobenzidine (DAB) staining, rice leaves were immersed in $1 \mathrm{mg} \mathrm{mL}^{-1} \mathrm{DAB}$ in $50 \mathrm{mM}$ Tris-acetate buffer ( $\mathrm{pH}$ 5.0) for $12 \mathrm{~h}$ at room temperature in the dark. To detect superoxide radical $\left(\mathrm{O}_{2}^{--}\right)$, leaves were incubated in $1 \mathrm{mg} \mathrm{mL}^{-1}$ nitro-blue tetrazolium (NBT) in a $10 \mathrm{mM}$ potassium phosphate solution ( $\mathrm{pH} 7.8$ ) at $25^{\circ} \mathrm{C}$ for $12 \mathrm{~h}$ in the dark. Then, the leaves were de-stained with $95 \%$ ethanol for $10 \mathrm{~min}$ at $100{ }^{\circ} \mathrm{C}$. De-staining was repeated several times until the chlorophyll was completely removed.

WT and OsWRKY55-OE (OE-1) internodes were immobilized in paraffin and sectioned as described previously [48]. In brief, the uppermost internodes of the main culms of the WT and OsWRKY55-OE (OE-1) plants were longitudinally sectioned, fixed in formaldehyde/glacial acetic acid/70\% ethanol (1:1:18, v/v/v) for $48 \mathrm{~h}$, and softened with 15\% hydrofluoric acid for 2 weeks. Then, the samples were dehydrated using a graded ethanol series. The dehydrated samples were embedded in paraffin (Sigma-Aldrich) and microsectioned to a thickness of $8 \mu \mathrm{m}$ using a microtome. The sections were stained with toluidine blue and viewed under a light microscope after paraffin removal with xylene.

\subsection{Accession Numbers}

Sequence data from this article can be found in the MSU Rice Genome Annotation Project (http: / / rice.plantbiology.msu.edu/analyses_search_locus.shtml, accessed on 18 April, 2020) databases under the following accession numbers: OsWRKY55/OsWRKY31 (LOC_Os03g20550), OsMPK7 (LOC_Os05g49140), OsMPK9 (LOC_Os05g50560), OsMPK201 (LOC_Os01g43910), OsMPK20-4 (LOC_Os01g47530), OsMPK3 (LOC_Os03g17700), OsMPK4-1 (LOC_Os10g38950), OsMPK6 (LOC_Os06g06090), OsMPK14 (LOC_Os02g05480), OsMPK16 (LOC_Os11g17080), OsMPK17-1 (LOC_Os06g49430), OsMPK17-2 (LOC_Os02g04230), OsMPK20-3 (LOC_Os06g26340), OsMPK4-2 (LOC_Os06g48590), OsMPK21-1 (LOC_Os05g50120), OsMPK21-2 (LOC_Os01g45620), OsActin (LOC_Os03g50885), Ubiquitin5 (LOC_Os01g22490), and OsAP2-39 (LOC_Os04g52090).

Supplementary Materials: The following are available online at https:/ / www.mdpi.com/article/10 .3390/ijms22094337/s1.

Author Contributions: X.D. and W.J. designed the study; K.H., Z.M., H.C., M.Z. and H.B. executed the experiments; Z.L. analyzed the data; K.H. and T.W. wrote the manuscript; X.D., W.J. and M.B. modified the manuscript. All authors have read and agreed to the published version of the manuscript.

Funding: This research was supported by National Natural Science Foundation of China (31701396 and 31901434), Science and Technology Development Plan of Jilin Province, China (20160204007NY), and Jilin Provincial and School Co-construction project (No. SXGJXX2019-2).

Institutional Review Board Statement: Not applicable.

Informed Consent Statement: Not applicable.

Conflicts of Interest: The authors declare no conflict of interest. 


\section{References}

1. $\quad$ Fang, Y.J.; Liao, K.F.; Du, H.; Xu, Y.; Song, H.Z.; Li, X.H.; Xiong, L.Z. A stress-responsive NAC transcription factor SNAC3 confers heat and drought tolerance through modulation of reactive oxygen species in rice. J. Exp. Bot. 2015, 66, 6803-6817. [CrossRef] [PubMed]

2. Xiong, L.M.; Schumaker, K.S.; Zhu, J.K. Cell signaling during cold, drought, and salt stress. Plant Cell 2002, 14, S165-S183. [CrossRef] [PubMed]

3. Yamaguchi-Shinozaki, K.; Shinozaki, K. Transcriptional regulatory networks in cellular responses and tolerance to dehydration and cold stresses. Annu. Rev. Plant Biol. 2006, 57, 781-803. [CrossRef]

4. Tang, Y.H.; Bao, X.X.; Zhi, Y.L.; Wu, Q.; Guo, Y.; Yin, X.H.; Zeng, L.Q.; Li, J.; Zhang, J.; He, W.L.; et al. Overexpression of a MYB family gene, $O s M Y B 6$, increases drought and salinity stress tolerance in transgenic rice. Front. Plant Sci. 2019, 10, 168. [CrossRef] [PubMed]

5. Seo, J.S.; Joo, J.; Kim, M.J.; Kim, Y.K.; Nahm, B.H.; Song, S.I.; Cheong, J.J.; Lee, J.S.; Kim, J.K.; Choi, Y.D. OsbHLH148, a basic helix-loop-helix protein, interacts with OsJAZ proteins in a jasmonate signaling pathway leading to drought tolerance in rice. Plant J. 2011, 65, 907-921. [CrossRef] [PubMed]

6. Oh, S.-J.; Kim, Y.S.; Kwon, C.-W.; Park, H.K.; Jeong, J.S.; Kim, J.-K. Overexpression of the transcription factor AP37 in rice improves grain yield under drought conditions. Plant Physiol. 2009, 150, 1368-1379. [CrossRef] [PubMed]

7. Yang, S.Q.; Xu, K.; Chen, S.J.; Li, T.F.; Xia, H.; Chen, L.; Liu, H.Y.; Luo, L.J. A stress-responsive bZIP transcription factor OsbZIP62 improves drought and oxidative tolerance in rice. BMC Plant Biol. 2019, 19, 260. [CrossRef]

8. Singh, D.; Laxmi, A. Transcriptional regulation of drought response: A tortuous network of transcriptional factors. Front. Plant Sci. 2015, 6, 895. [CrossRef]

9. Eulgem, T.; Rushton, P.J.; Robatzek, S.; Somssich, I.E. The WRKY superfamily of plant transcription factors. Trends Plant Sci. 2000, 5, 199-206. [CrossRef]

10. Chujo, T.; Takai, R.; Akimoto-Tomiyama, C.; Ando, S.; Minami, E.; Nagamura, Y.; Kaku, H.; Shibuya, N.; Yasuda, M.; Nakashita, $\mathrm{H}$;; et al. Involvement of the elicitor-induced gene OsWRKY53 in the expression of defense-related genes in rice. Biochim. Biophys. Acta 2007, 1769, 497-505. [CrossRef]

11. Liu, X.Q.; Bai, X.J.; Wang, X.J.; Chu, C.C. OsWRKY71, a rice transcription factor, is involved in rice defense response. J. Plant Physiol. 2007, 164, 969-979. [CrossRef] [PubMed]

12. Wang, H.H.; Hao, J.J.; Chen, X.J.; Hao, Z.N.; Wang, X.; Lou, Y.G.; Peng, Y.L.; Guo, Z.J. Overexpression of rice WRKY89 enhances ultraviolet B tolerance and disease resistance in rice plants. Plant Mol. Biol. 2007, 65, 799-815. [CrossRef] [PubMed]

13. Peng, Y.; Bartley, L.E.; Chen, X.W.; Dardick, C.; Chern, M.; Ruan, R.; Canlas, P.E.; Ronald, P.C. OsWRKY62 is a negative regulator of basal and Xa21-mediated defense against Xanthomonas oryzae pv. oryzae in rice. Mol. Plant 2008, 1, 446-458. [CrossRef] [PubMed]

14. Wang, C.T.; Ru, J.N.; Liu, Y.W.; Li, M.; Zhao, D.; Yang, J.F.; Fu, J.D.; Xu, Z.S. Maize WRKY transcription factor ZmWRKY106 confers drought and heat tolerance in transgenic plants. Int. J. Mol. Sci. 2018, 19, 3046. [CrossRef]

15. Wang, C.T.; Ru, J.N.; Liu, Y.W.; Yang, J.F.; Li, M.; Xu, Z.S.; Fu, J.D. The Maize WRKY transcription factor ZmWRKY40 confers drought resistance in transgenic Arabidopsis. Int. J. Mol. Sci. 2018, 19, 2580. [CrossRef]

16. Shi, W.Y.; Du, Y.T.; Ma, J.; Min, D.H.; Jin, L.G.; Chen, J.; Chen, M.; Zhou, Y.B.; Ma, Y.Z.; Xu, Z.S.; et al. The WRKY transcription factor GmWRKY12 confers drought and salt tolerance in Soybean. Int. J. Mol. Sci. 2018, 19, 4087. [CrossRef]

17. Wei, W.; Liang, D.W.; Bian, X.H.; Shen, M.; Xiao, J.H.; Zhang, W.K.; Ma, B.; Lin, Q.; Lv, J.; Chen, X.; et al. GmWRKY54 improves drought tolerance through activating genes in abscisic acid and $\mathrm{Ca}^{2+}$ signaling pathways in transgenic soybean. Plant J. 2019, 100, 384-398. [CrossRef]

18. Yokotani, N.; Sato, Y.; Tanabe, S.; Chujo, T.; Shimizu, T.; Okada, K.; Yamane, H.; Shimono, M.; Sugano, S.; Takatsuji, H.; et al. WRKY76 is a rice transcriptional repressor playing opposite roles in blast disease resistance and cold stress tolerance. J. Exp. Bot. 2013, 64, 5085-5097. [CrossRef]

19. Ma, H.G.; Chen, J.; Zhang, Z.Z.; Ma, L.; Yang, Z.Y.; Zhang, Q.L.; Li, X.H.; Xiao, J.H.; Wang, S.P. MAPK kinase 10.2 promotes disease resistance and drought tolerance by activating different MAPKs in rice. Plant J. 2017, 92, 557-570. [CrossRef]

20. Asai, T.; Tena, G.; Plotnikova, J.; Willmann, M.R.; Chiu, W.L.; Gomez-Gomez, L.; Boller, T.; Ausubel, F.M.; Sheen, J. MAP kinase signalling cascade in Arabidopsis innate immunity. Nature 2002, 415, 977-983. [CrossRef]

21. Andreasson, E.; Jenkins, T.; Brodersen, P.; Thorgrimsen, S.; Petersen, N.H.; Zhu, S.; Qiu, J.L.; Micheelsen, P.; Rocher, A.; Petersen, M.; et al. The MAP kinase substrate MKS1 is a regulator of plant defense responses. EMBO J. 2005, 24, 2579-2589. [CrossRef]

22. Shen, H.S.; Liu, C.T.; Zhang, Y.; Meng, X.P.; Zhou, X.; Chu, C.C.; Wang, X.P. OsWRKY30 is activated by MAP kinases to confer drought tolerance in rice. Plant Mol. Biol. 2012, 80, 241-253. [CrossRef]

23. Koo, S.C.; Moon, B.C.; Kim, J.K.; Kim, C.Y.; Sung, S.J.; Kim, M.C.; Cho, M.J.; Cheong, Y.H. OsBWMK1 mediates SA-dependent defense responses by activating the transcription factor OsWRKY33. Biochem. Biophys. Res. Commun. 2009, 387, 365-370. [CrossRef]

24. Hu, L.F.; Ye, M.; Li, R.; Zhang, T.F.; Zhou, G.X.; Wang, Q.; Lu, J.; Lou, Y.G. The rice transcription factor WRKY53 suppresses herbivore-induced defenses by acting as a negative feedback modulator of mitogen-activated protein kinase activity. Plant Physiol. 2015, 169, 2907-2921. [CrossRef]

25. Zhou, C.L.; Lin, Q.B.; Lan, J.; Zhang, T.Y.; Liu, X.; Miao, R.; Mou, C.L.; Nguyen, T.; Wang, J.C.; Zhang, X.; et al. WRKY transcription factor OsWRKY29 represses seed dormancy in rice by weakening abscisic acid response. Front. Plant Sci. 2020, 11, 691. [CrossRef] 
26. Ding, Z.J.; Yan, J.Y.; Li, G.X.; Wu, Z.C.; Zhang, S.Q.; Zheng, S.J. WRKY41 controls Arabidopsis seed dormancy via direct regulation of $A B I 3$ transcript levels not downstream of ABA. Plant J. 2014, 79, 810-823. [CrossRef]

27. Jing, S.J.; Zhou, X.; Song, Y.; Yu, D.Q. Heterologous expression of OsWRKY23 gene enhances pathogen defense and dark-induced leaf senescence in Arabidopsis. Plant Growth Regul. 2009, 58, 181-190. [CrossRef]

28. Miao, Y.; Laun, T.; Zimmermann, P.; Zentgraf, U. Targets of the WRKY53 transcription factor and its role during leaf senescence in Arabidopsis. Plant Mol. Biol. 2004, 55, 853-867. [CrossRef]

29. Ulker, B.; Mukhtar, M.S.; Somssich, I.E. The WRKY70 transcription factor of Arabidopsis influences both the plant senescence and defense signaling pathways. Planta 2007, 226, 125-137. [CrossRef]

30. Cai, Y.H.; Chen, X.J.; Xie, K.; Xing, Q.K.; Wu, Y.W.; Li, J.; Du, C.H.; Sun, Z.X.; Guo, Z.J. Dlf1, a WRKY transcription factor, is involved in the control of flowering time and plant height in rice. PLoS ONE 2014, 9, e102529. [CrossRef]

31. Xie, Z.; Zhang, Z.L.; Zou, X.; Huang, J.; Ruas, P.; Thompson, D.; Shen, Q.J. Annotations and functional analyses of the rice WRKY gene superfamily reveal positive and negative regulators of abscisic acid signaling in aleurone cells. Plant Physiol. 2005, 137, 176-189. [CrossRef] [PubMed]

32. Zhang, J.; Peng, Y.L.; Guo, Z.J. Constitutive expression of pathogen-inducible OsWRKY31 enhances disease resistance and affects root growth and auxin response in transgenic rice plants. Cell Res. 2008, 18, 508-521. [CrossRef] [PubMed]

33. Wu, T.; Zhang, M.X.; Zhang, H.J.; Huang, K.; Chen, M.J.; Chen, C.; Yang, X.; Li, Z.; Chen, H.Y.; Ma, Z.M.; et al. Identification and characterization of EDT1 conferring drought tolerance in rice. J. Plant Biol. 2019, 62, 39-47. [CrossRef]

34. Hamel, L.P.; Nicole, M.C.; Sritubtim, S.; Morency, M.J.; Ellis, M.; Ehlting, J.; Beaudoin, N.; Barbazuk, B.; Klessig, D.; Lee, J.; et al Ancient signals: Comparative genomics of plant MAPK and MAPKK gene families. Trends Plant Sci. 2006, 11, 192-198. [CrossRef]

35. Yaish, M.W.; El-Kereamy, A.; Zhu, T.; Beatty, P.H.; Good, A.G.; Bi, Y.M.; Rothstein, S.J. The APETALA-2-like transcription factor OsAP2-39 controls key interactions between abscisic acid and gibberellin in rice. PLoS Genet. 2010, 6, e1001098. [CrossRef]

36. Rushton, P.J.; Somssich, I.E.; Ringler, P.; Shen, Q.J. WRKY transcription factors. Trends Plant Sci. 2010, 15, 247-258. [CrossRef]

37. Xu, M.; Zhu, L.; Shou, H.X.; Wu, P. A PIN1 family gene, OsPIN1, involved in auxin-dependent adventitious root emergence and tillering in rice. Plant Cell Physiol. 2005, 46, 1674-1681. [CrossRef]

38. Kim, C.-Y.; Vo, K.T.X.; Nguyen, C.D.; Jeong, D.-H.; Lee, S.-K.; Kumar, M.; Kim, S.-R.; Park, S.-H.; Kim, J.-K.; Jeon, J.-S. Functional analysis of a cold-responsive rice WRKY gene, OsWRKY71. Plant Biotechnol. Rep. 2016, 10, 13-23. [CrossRef]

39. Deeba, F.; Sultana, T.; Mahmood, T.; O'Shea, C.; Skriver, K.; Naqvi, S.M.S. Involvement of WRKY, MYB and DOF DNA-binding proteins in interaction with a rice germin-like protein gene promoter. Acta Physiol. Plant. 2017, 39. [CrossRef]

40. Zhang, Z.Y.; Li, J.H.; Li, F.; Liu, H.H.; Yang, W.S.; Chong, K.; Xu, Y.Y. OsMAPK3 phosphorylates OsbHLH002/OsICE1 and inhibits its ubiquitination to activate OsTPP1 and enhances rice chilling tolerance. Dev. Cell 2017, 43, 731-743.e5. [CrossRef]

41. Zhang, Z.Y.; Liu, H.H.; Sun, C.; Ma, Q.B.; Bu, H.Y.; Chong, K.; Xu, Y.Y. A C2H2 zinc-finger protein OsZFP213 interacts with OsMAPK3 to enhance salt tolerance in rice. J. Plant Physiol. 2018, 229, 100-110. [CrossRef]

42. Moustafa, K.; AbuQamar, S.; Jarrar, M.; Al-Rajab, A.J.; Trémouillaux-Guiller, J. MAPK cascades and major abiotic stresses. Plant Cell Rep. 2014, 33, 1217-1225. [CrossRef]

43. Ueno, Y.; Yoshida, R.; Kishi-Kaboshi, M.; Matsushita, A.; Jiang, C.J.; Goto, S.; Takahashi, A.; Hirochika, H.; Takatsuji, H. Abiotic stresses antagonize the rice defence pathway through the tyrosine-dephosphorylation of OsMPK6. PLoS Pathog. 2015, 11, e1005231. [CrossRef]

44. Wan, L.Y.; Zhang, J.F.; Zhang, H.W.; Zhang, Z.J.; Quan, R.D.; Zhou, S.R.; Huang, R.F. Transcriptional activation of OsDERF1 in OsERF3 and OsAP2-39 negatively modulates ethylene synthesis and drought tolerance in rice. PLoS ONE 2011, 6, e25216. [CrossRef]

45. Livak, K.J.; Schmittgen, T.D. Analysis of relative gene expression data using real-time quantitative PCR. Methods 2002, 25, 402-408. [CrossRef]

46. Guo, X.L.; Hou, X.M.; Fang, J.; Wei, P.W.; Xu, B.; Chen, M.L.; Feng, Y.Q.; Chu, C.C. The rice GERMINATION DEFECTIVE 1, encoding a B3 domain transcriptional repressor, regulates seed germination and seedling development by integrating GA and carbohydrate metabolism. Plant J. 2013, 75, 403-416. [CrossRef]

47. Yoo, S.D.; Cho, Y.H.; Sheen, J. Arabidopsis mesophyll protoplasts: A versatile cell system for transient gene expression analysis. Nat. Protoc. 2007, 2, 1565-1572. [CrossRef]

48. Ma, Y.M.; Yang, C.; He, Y.; Tian, Z.H.; Li, J.X. Rice OVATE family protein 6 regulates plant development and confers resistance to drought and cold stresses. J. Exp. Bot. 2017, 68, 4885-4898. [CrossRef] 